\title{
Low-Threshold Primary Afferent Drive onto GABAergic Interneurons in the Superficial Dorsal Horn of the Mouse
}

\author{
Claire A. Daniele ${ }^{1}$ and Amy B. MacDermott ${ }^{1,2}$ \\ Departments of ${ }^{1}$ Neuroscience and ${ }^{2}$ Physiology and Cellular Biophysics, Columbia University, New York, New York 10032
}

\begin{abstract}
Inhibition in the spinal cord dorsal horn is crucial for maintaining separation of touch and pain modalities. Disruption of this inhibition results in allodynia, allowing low-threshold drive onto pain and temperature-sensitive projection neurons. This low-threshold (LT) excitatory pathway is normally under strong inhibition. We hypothesized that superficial dorsal horn inhibitory neurons, which would be ideally located to suppress LT drive onto projection neurons in a feedforward manner, are driven by LT input. In addition, because disinhibition-induced allodynia shares some features with the immature dorsal horn such as elevated sensitivity to LT input, we also questioned whether LT drive onto inhibitory neurons changes during postnatal maturation. To investigate these questions, slices were made at different ages from transgenic mice with enhanced green fluorescent protein expression in GABAergic neurons and whole-cell recordings were made from these fluorescent neurons. Evoked synaptic activity was measured in response to electrical stimulation of the dorsal root. We demonstrate that $\mathrm{A} \beta$ fibers activate a significant proportion of superficial dorsal horn GABAergic neurons. This occurs with similar excitatory synaptic drive throughout postnatal maturation, but with a greater prevalence at younger ages. These GABAergic neurons are well situated to contribute to suppressing LT activation of output projection neurons. In addition, the majority of these GABAergic neurons also had convergent input from high-threshold fibers, suggesting that this novel subclass of GABAergic neurons is important for gating innocuous as well as noxious information.
\end{abstract}

Key words: spinal cord; inhibition; pain; development; afferent; substantia gelatinosa

\section{Introduction}

Local inhibition in the spinal cord dorsal horn is essential for appropriate encoding of sensory signals. This, in turn, is dependent upon proper presynaptic and postsynaptic connectivity. Inhibitory neurons represent a significant percentage $(\sim 30 \%)$ of the total population of neurons in laminae I-III (Todd and Sullivan, 1990). Superficial dorsal horn (SDH) (lamina I and II) inhibitory neurons receive $\mathrm{A} \delta$ and C-fiber monosynaptic or polysynaptic drive (Lu and Perl, 2003; Hantman et al., 2004; Heinke et al., 2004), suggesting a regulatory role in high-threshold (HT) sensory signaling such as pain or temperature.

Interestingly, at least two roles have been predicted for lowthreshold (LT) drive of inhibitory neurons in SDH. The first role, predicted by the gate control theory, is to mediate the suppression of pain by peripheral touch, as when rubbing near an injured area leads to temporary relief (Melzack and Wall, 1965). The second is feedforward inhibition in the context of neuropathic or inflammatory pain. This is predicted because pharmacological disruption of inhibition in vivo with intrathecal antagonists to $\mathrm{GABA}_{\mathrm{A}}$ or glycine receptors causes pain behaviors in response to

Received 0ct. 23, 2008; revised Nov. 25, 2008; accepted Nov. 29, 2008.

This work was supported by National Institutes of Health Grant NS029797. We thank Dr. Carole Torsney for helpful discussion and critical reading of this manuscript, Lillian Tien for help with the confocal imaging, and Dr. Papiya Choudhury for excellent technical assistance.

Correspondence should be addressed to Claire A. Daniele, Department of Neuroscience, Columbia University, 630 West 168th Street, New York, NY 10032. E-mail: cad94@columbia.edu.

D01:10.1523/JNEUROSCI.5120-08.2009

Copyright $\odot 2009$ Society for Neuroscience $\quad$ 0270-6474/09/290686-10\$15.00/0 touch (allodynia) (Beyer et al., 1985; Roberts et al., 1986; Yaksh, 1989). Consistent with this, disinhibition in vitro strongly enhances low-threshold ( $\mathrm{A} \beta$ fiber) activation of lamina II neurons (Baba et al., 2003). Furthermore, nociceptive lamina I projection neurons develop novel $\mathrm{A} \beta$ fiber activation during disinhibition in vitro (Torsney and MacDermott, 2006) as well as in vivo (Keller et al., 2007). These results demonstrate the presence of a fully wired, excitatory, polysynaptic pathway between low-threshold afferents and nociceptive projection neurons that is normally suppressed by inhibition. Suppression of this excitatory pathway could occur through feedforward $\mathrm{A} \beta$ fiber drive of local inhibitory neurons.

Disinhibition-induced allodynia shares some features with the developing spinal cord, which is characterized by greater sensitivity to low-threshold cutaneous stimuli (Fitzgerald, 1985; Torsney and Fitzgerald, 2002) and minimal inhibition in SDH (Baccei and Fitzgerald, 2004; Cordero-Erausquin et al., 2005). It was proposed that $\mathrm{A} \beta$ fibers terminate more superficially in the dorsal horn of newborns and this is supported by morphological studies showing gradual withdrawal to deeper layers with postnatal maturation (Fitzgerald et al., 1994; Fitzgerald, 2005). This view was challenged by recent characterization of single cutaneous A $\beta$ fibers (Woodbury et al., 2001; Woodbury and Koerber, 2007). Independent of anatomical findings, however, physiological studies report a decreasing number of lamina II neurons that are activated by $\mathrm{A} \beta$ fibers during maturation (Jennings and Fitzgerald, 1998; Park et al., 1999; Nakatsuka et al., 2000). Therefore, the prevalence of potential $A \beta$ fiber activation of inhibitory 
neurons in the SDH may also undergo changes during maturation.

Here, we show that $\mathrm{A} \beta$ fibers activate a significant proportion of SDH GABAergic neurons with a similar magnitude throughout postnatal development, but a greater prevalence at younger ages. These data reveal a novel subclass of intrinsic GABAergic neurons that may be involved in the gating of innocuous, as well as nociceptive information.

\section{Materials and Methods}

Spinal cord slice and dorsal root preparation. Mice used in this study were homozygous for a transgene that has enhanced green fluorescent protein (EGFP) expression driven by the mouse gadl gene promoter. This gene encodes for GAD67 (Oliva et al., 2000), a $67 \mathrm{kDa}$ isoform of the enzyme required to produce GABA (Kaufman et al., 1986; Erlander et al., 1991). Breeding pairs were obtained from The Jackson Laboratory and interbred at our facility. Nearly all of these fluorescent neurons are GABAergic in lamina I and II of the spinal cord (Oliva et al., 2000; Heinke et al., 2004; Dougherty et al., 2005).

Postnatal day 7-11 (P7-P11), P17-P24, and P30-P45 mice were anesthetized with isoflurane, decapitated and the lumbar (L3-L5) segments of the spinal cord were removed in a manner approved by the Columbia University Institutional Animal Care and Use Committee. Vertebrae with spinal cords were placed in ice-cold dissection Krebs solution (in mм: $95 \mathrm{NaCl}, 2.5 \mathrm{KCl}, 26 \mathrm{NaHCO}_{3}, 1.25 \mathrm{NaH}_{2} \mathrm{PO}_{4} \mathrm{H}_{2} \mathrm{O}, 6 \mathrm{MgCl}_{2}, 1.5$ $\mathrm{CaCl}_{2}, 25$ glucose, 50 sucrose, 1 kynurenic acid), saturated with $95 \% \mathrm{O}_{2} /$ $5 \% \mathrm{CO}_{2}$. The L3-L5 segments were removed and embedded in agarose and glued to the vibratome with the spinal cord midline parallel to the blade. Alternatively, sometimes the spinal cord was glued to the vibratome specimen plate at a slightly tilted angle, with the ventral horn slightly higher on one side to facilitate sectioning. 350-500 $\mu \mathrm{m}$ parasagittal slices with dorsal roots attached were cut using a vibratome (Leica), and allowed to recover in $95 \% \mathrm{O}_{2} / 5 \% \mathrm{CO}_{2}$ saturated recovery Krebs (same as dissection Krebs, but lacking kynurenic acid) at $37^{\circ} \mathrm{C}$ for $1 \mathrm{~h}$ and then at room temperature until use. Alternatively, for compound action potential recordings, only the L3-L5 dorsal roots were isolated, and were stored in the same solution as the slices at $37^{\circ} \mathrm{C}$ for $1 \mathrm{~h}$ and then at room temperature until use.

We limited this study to recordings from parasagittal slices because when recordings were made from transverse slices, there was an agedependent change in the percentage of neurons with no detectable input (our unpublished observations). Presumably, the transverse slice preparation caused damage to the incoming afferents along their rostrocaudal trajectory or to the pathways they activate in the dorsal horn. Each parasagittal slice or dorsal root was placed into a perfusion chamber with a constant flow of $95 \% \mathrm{O}_{2} / 5 \% \mathrm{CO}_{2}$ saturated bath Krebs (in mM: $125 \mathrm{NaCl}$, $2.5 \mathrm{KCl}, 26 \mathrm{NaHCO}_{3}, 1.25 \mathrm{NaH}_{2} \mathrm{PO}_{4} \mathrm{H}_{2} \mathrm{O}, 1 \mathrm{MgCl}_{2}, 2 \mathrm{CaCl}_{2}, 25$ glucose) at room temperature.

Patch-clamp recording. Dorsal horn neurons were viewed using an upright microscope with a $40 \times$ water-immersion objective, infrared differential interference contrast (IR-DIC) and fluorescence. Whole-cell patch-clamp recordings were made from GABAergic EGFP ${ }^{+}$neurons. Internal solution (in $\mathrm{mM}$ ) was as follows: $120 \mathrm{~K}$ methane-sulfonate, 10 $\mathrm{NaCl}, 1 \mathrm{CaCl}_{2}, 10$ EGTA, 10 Hepes, $5 \mathrm{Mg}$ ATP, $0.5 \mathrm{Na}$ GTP, $0.1 \%$ biocytin; or $100 \mathrm{Cs}$ methanesulfonate, $10 \mathrm{Na}$ methanesulfonate, $5 \mathrm{QX} 314-\mathrm{Cl}$, 10 TEA Cl, $1 \mathrm{CaCl}_{2}, 10$ EGTA, 10 Hepes, $5 \mathrm{Mg}$ ATP, $0.5 \mathrm{Na}$ GTP, $0.1 \%$ biocytin. Solutions were brought to a $\mathrm{pH}$ of 7.2. Data were acquired with pClamp 9 (Molecular Devices) using an Axopatch 200B amplifier (Molecular Devices), filtered at $5 \mathrm{kHz}$, and digitized at $50 \mathrm{kHz}$. Before recording, the junction potential was corrected.

For neurons from which action potentials were recorded, the resting membrane potential was noted immediately after establishing whole-cell configuration. Then firing patterns were assessed using $1 \mathrm{~s}$ graded depolarizing current steps in current clamp. Data from neurons with resting membrane potentials positive to $-50 \mathrm{mV}$ were discarded. Under IR-DIC optics, lamina II appears translucent. After the recording, if the cell body of the neuron was within this translucent area, it was judged to be in lamina II. If it was above or just adjacent to the translucent band, it was considered to be in lamina I.

Extracellular compound action potential recording from dorsal roots. Suction electrodes for stimulating and recording were placed at either end of the dorsal root. Stimuli were delivered using a stimulus isolator (ISO Flex Stimulus isolator, A.M.P.I.) and action potential volleys were acquired using an extracellular differential amplifier (Cornerstone, by Dagan). The stimulation intensities used were (in $\mu \mathrm{A}$ ) 4, 8, 12, 16, 20, 25, 30-90 (in increments of 10, and 100-300 (in increments of 25), all at 0.2 $\mathrm{Hz}$. Five sweeps were recorded per stimulus intensity.

Responses to dorsal root stimulation recorded in voltage clamp to assess primary afferent input onto dorsal horn neurons. Stimulation intensities used in our voltage-clamp experiments were $4,8,12,16,20,25 \mu \mathrm{A}$ for A $\beta$ fibers, 50 and $100 \mu \mathrm{A}$ for $\mathrm{A} \delta$ fibers, and 300 and $500 \mu \mathrm{A}$ for C fibers (ISO Flex Stimulus isolator, A.M.P.I.). These values were based on measurements made from mouse dorsal roots. These had similar threshold stimulus intensity ranges to those previously reported in rat (Nakatsuka et al., 2000). Three consecutive synaptic responses were recorded at low frequency for each stimulus intensity $(0.05 \mathrm{~Hz}, 0.1 \mathrm{~ms}$ duration $)$. C-fiber intensities were sometimes tested at both 0.1 , and $0.5 \mathrm{~ms}$ duration. The minimal stimulus intensity needed to evoke an $\mathrm{A} \beta$ fiber response was noted when the response first appeared. Often, the response amplitude grew larger as the stimulation intensity was increased. There were a few cases where intensities between 12 and $25 \mu \mathrm{A}$ were not tested and such cells with $\mathrm{A} \beta$ fiber input were not included in the statistical analysis of the minimal stimulus intensities for $A \beta$ fiber-evoked currents. One-way ANOVA followed by Newman-Keuls posttests were used to compare the minimal stimulus intensities required to evoke $A \beta$ fiber responses among different ages (Prism 4.0, GraphPad Software).

Stable responses time-locked to the stimulus were classified as monosynaptic or polysynaptic based on their ability to follow high-frequency stimulation (Nakatsuka et al., 2000; Torsney and MacDermott, 2006). However, because $\mathrm{A} \delta$ fibers begin to fail firing action potentials at stimulation frequencies $>2 \mathrm{~Hz}$, and $\mathrm{C}$ fibers fail at frequencies $>1 \mathrm{~Hz}$ (Nakatsuka et al., 2000), we were limited in the stimulation frequencies we could test. Thus, $\mathrm{A} \beta$ fiber mediated responses were tested at $20 \mathrm{~Hz}$; $\mathrm{A} \delta$ responses at $2 \mathrm{~Hz}$; and $\mathrm{C}$-fiber responses at $1 \mathrm{~Hz}$. $\mathrm{A} \beta$ or $\mathrm{A} \delta$ fiber responses with a lack of failures as well as consistent onset latency on consecutive trials at high frequency were considered to be monosynaptic. If the onset latency varied $>2 \mathrm{~ms}$ and/or there were failures, the response was determined to be polysynaptic. C-fiber responses with a lack of failures were considered to be monosynaptic, even with variable latency (Nakatsuka et al., 2000).

Analysis. Separate components of the compound action potentials could be distinguished, each having a tri-phasic profile. The first peak is a prominent positive potential, followed by a negative peak and another smaller positive peak. The amplitude of each component of the compound action potential was calculated by measuring the distance between the first positive peak and the negative peak. Conduction velocities were estimated by calculating the distance between the stimulating and recording electrodes divided by the latency of the negative peak, for each component of the compound action potential. One-way ANOVA followed by Newman-Keuls posttests were used to compare the conduction velocities of $\mathrm{A} \alpha / \mathrm{A} \beta$ component of the compound action potentials among different ages (Prism 4.0, GraphPad Software).

To determine the magnitude of afferent evoked synaptic responses, the area under the curve (the integrated current) was measured from the stimulus artifact to the end of the trace (500 ms), using Clampfit 9 Software (Molecular Devices). One-way ANOVA followed by NewmanKeuls posttests were used to compare the averaged peak current and integrated current among different ages (Prism 4.0, GraphPad Software). To obtain high-threshold response magnitude for neurons with convergent input (for example, from $\mathrm{A} \beta$ and $\mathrm{C}$ fibers), we subtracted the integrated current of response recorded at $25 \mu \mathrm{A}$ from the integrated current of the maximal high-threshold response, before calculating the mean for each age group. In the rare cases where unclamped spikes were observed in the responses, those cells were excluded from the integrated current analysis.

The $\chi^{2}$ test for trend was used to calculate the statistical significance of 
the differences in prevalence of the major types of afferent input at different ages (Prism 4.0, GraphPad Software). Fisher's exact test was used to compare the prevalence of $A \beta$ fiber input between lamina I and II, and to compare the prevalence of monosynaptic $A \beta$ fiber input across ages (GraphPad Software).

Immunocytochemistry. After the recording, some slices were fixed in $4 \%$ paraformaldehyde in $0.1 \mathrm{M}$ phosphate buffer for $24-48 \mathrm{~h}$ at $4^{\circ} \mathrm{C}$. Then these were washed with phosphate buffered saline (PBS) $3 \times 5$ min each. All subsequent incubations were performed with gentle agitation on an orbital shaker. Slices were immersed in blocking solution $(0.1 \%$ Triton $\mathrm{X}-100,10 \%$ normal goat serum (NGS) in PBS) for $1 \mathrm{~h}$ at room temperature; and then incubated with rabbit antiserum to $\mathrm{PKC} \gamma(1: 1000$ in blocking solution, Santa Cruz Biotechnology) overnight at room temperature. After this, slices were rinsed $4 \times$ in PBS, and then incubated in secondary antibody diluent (1\% BSA, $0.1 \%$ Triton X-100 and $10 \%$ NGS in PBS) for $1 \mathrm{~h}$ at room temperature before incubation with streptavidin-Cy5 (1:500, Zymed/Invitrogen) and Cy3-goat anti-rabbit IgG (1:800, Jackson Immuno Research), or streptavidin-Alexa 568 (1:500, Zymed/Invitrogen) and Cy5-goat antirabbit IgG (1:500, Jackson Immuno Research) for $3 \mathrm{~h}$ at room temperature. Finally these were washed in PBS $3 \times$, mounted on slides and fluorescent images were captured with a confocal microscope (Nikon). Further analysis of $z$-stacks was performed using Nikon EZ-C1 viewer software and Adobe Photoshop, where pseudo-color was used and brightness and contrast adjusted.

\section{Results}

A $\boldsymbol{\beta}$ fibers activate a significant proportion of GABAergic neurons in lamina I and II

To address whether $A \beta$ fibers activate inhibitory neurons, we recorded from preidentified GABAergic neurons expressing EGFP in lamina I and II of spinal cord slices. Fluorescent and transmitted light images from an acute slice of spinal cord are shown in Figure 1, $A$ and $B$, respectively. Lamina II can be distinguished from other laminae based on the translucent appearance of that cell layer under transmitted light (Fig. $1 B$ ). A neuron was judged to be in lamina I if its cell body was above or on the dorsal border of the translucent region and in lamina II if it was within that region. Many fluorescent neurons can be seen throughout laminae I-III (Fig. 1A,B). Although only $30 \%$ of GABAergic neurons are fluorescent in lamina I and II, EGFP labeled neurons are morphologically and physiologically diverse and numerous suggesting they represent many of the dorsal horn inhibitory neurons (Heinke et al., 2004; Dougherty et al., 2005).

Recordings were made from 40 fluorescent GABAergic neurons whose cell bodies were located in lamina I-II in parasagittal spinal cord slices taken from mice on postnatal days 30-40 (P30-40). These include 12 neurons in lamina I and 28 in lamina II. Figure $1 C$ depicts a schematic of the slice preparation, with a parasagittal slice outlined in red. This preparation allowed stimulation of the dorsal root (Fig. 1C, arrowhead) while postsynaptic responses were recorded from individual GABAergic neurons. Of the recorded neurons later processed for biocytin and costained with antibodies to PKC $\gamma$, all had cell bodies located either above or within the dense band of PKC $\gamma$ staining $(n=8)$, a marker that highlights inner lamina II (Malmberg et al., 1997; Polgar et al., 1999 ). Figure $1, D$ and $E$, shows examples of GABAergic neurons that were filled with biocytin (red), surrounded by other EGFP ${ }^{+}$ GABAergic neurons (green), and above or within the band of PKC $\gamma$ staining (blue). The neuron in Figure $1 D$ is located on the border between lamina I and II and has a morphology consistent with vertical cells (Grudt and Perl, 2002; Heinke et al., 2004), as has been observed among other EGFP ${ }^{+}$GABAergic neurons from this same transgenic mouse line (Heinke et al., 2004). We also noted islet cell morphology in other biocytin-filled EGFP ${ }^{+}$ neurons (data not shown), also consistent with previous observations (Bicknell and Beal, 1984; Grudt and Perl, 2002; Heinke et al., 2004).

Synaptic responses evoked by activation of LT primary afferent fibers ( $\mathrm{A} \beta$ fibers) were recorded from a subset of EGFP ${ }^{+}$ GABAergic neurons. Figure 2, $A$ and $B$, shows examples of LT synaptic responses from such a neuron. Synaptic currents were recorded in response to stimulation of the dorsal root at $\mathrm{A} \beta$ fiber intensity $(25 \mu \mathrm{A})$ and at low frequency $(0.05 \mathrm{~Hz})$ (Fig. $2 \mathrm{~A}$, arrow). Subsequently, each response was tested at a higher frequency $(20 \mathrm{~Hz})$ (Fig. $2 \mathrm{~B})$ to determine whether it was due to 

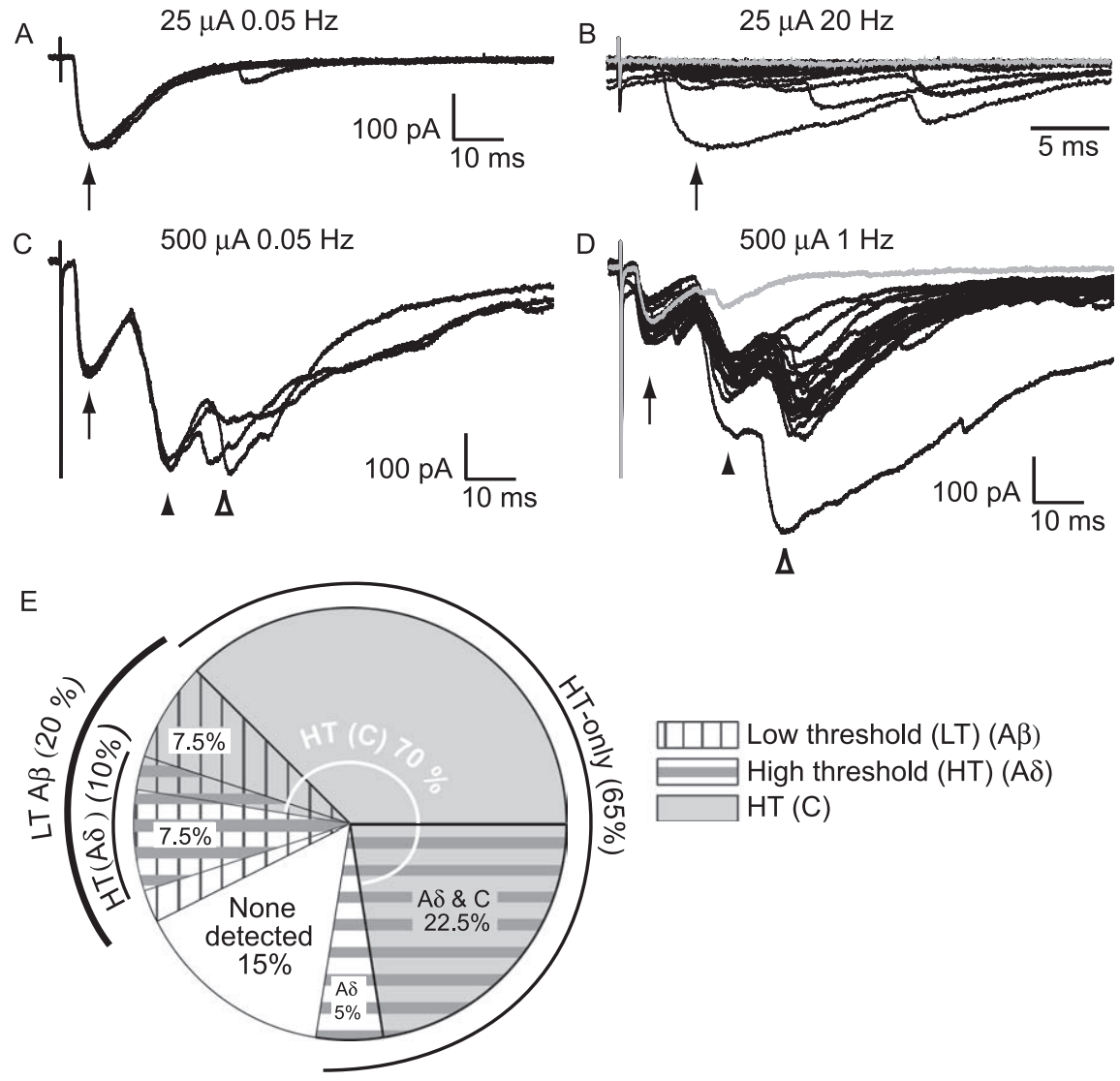

Figure 2. A subclass of EGFP-positive GABAergic neurons had low-threshold $(A \beta)$ fiber input as well as input from highthreshold fibers ( $A \delta$ and/or $C$ ). Shown here $(A-D)$ are example recordings from the same $P 35 \mathrm{GABAergic}$ neuron with input from $A \beta$ and ( fibers. $\boldsymbol{A}$, Three consecutive traces show responses to low-frequency $A \beta$ fiber stimulation $(0.05 \mathrm{~Hz}, 25 \mu \mathrm{A}$, arrow). $\boldsymbol{B}$, Twenty consecutive $A \beta$ fiber responses to high-frequency stimulation ( $20 \mathrm{~Hz}$, arrow, expanded timescale), included failures (gray trace), consistent with a polysynaptic input. These responses are dominated by cation current, given the holding potential and the expected chloride reversal potential. C, C-fiber input was observed when the stimulation intensity was increased (500 $\mu \mathrm{A}$, at low frequency, arrowheads). $\boldsymbol{D}$, The (-fiber response had a monosynaptic component with no failures (but note the small amplitude in the gray trace) when tested at high frequency $(1 \mathrm{~Hz}$, filled arrowhead). There was also a later, polysynaptic C-fiber component with failures (open arrowhead, illustrated by the gray trace). $\boldsymbol{E}$, The proportion of GABAergic neurons with input from different afferent fiber classes is summarized. For simplicity, this representation does not distinguish between monosynaptic and polysynaptic responses. There is considerable overlap between the subsets of GABAergic neurons receiving input from each class of afferent fiber type.

monosynaptic or polysynaptic input. This LT response was defined as polysynaptic because there were failures at high frequency (Fig. $2 B$, gray trace). In addition to LT synaptic drive, this neuron also had HT drive. When the stimulus intensity was increased to $500 \mu \mathrm{A}$, additional C-fiber responses were recorded (Fig. 2C, arrowheads). These included a monosynaptic component with no failures at high stimulation frequency (Fig. $2 D$, filled arrowhead) and a longer latency polysynaptic component with failures at high frequency (Fig. $2 D$, open arrowhead).

Figure $2 E$ is a summary of all of the afferent-evoked data. Distinct wedges in the pie chart represent the proportion of neurons with input from each type of afferent fiber. The overlap among these is considerable. Each proportion represents the total synaptic drive including both mono and polysynaptic components. A $\beta$ fibers synaptically excited $20 \%$ (8/40) of the EGFPlabeled GABAergic neurons in the dorsal horn (Fig. $2 E$, verticalhatched wedges). Most of this LT input was polysynaptic. Only one monosynaptic $\mathrm{A} \beta$ fiber input was observed, representing only $2.5 \%(1 / 40)$ of the GABAergic neurons.

We next determined whether $A \beta$ fiber synaptic input varied with laminar location. $A \beta$ fiber input was found in $17 \%(2 / 12)$ of lamina I, and $22 \%(6 / 28)$ of lamina II $\mathrm{EGFP}^{+}$neurons. These percentages were not significantly different $(p>0.05)$. Therefore, we conclude that this LT input can be found in $\mathrm{EGFP}^{+}$GABAergic neurons with similar prevalence in lamina I versus II.

Interestingly, the LT-driven GABAergic neurons described here were morphologically heterogeneous. Among those filled with biocytin and stained $(n=6)$, a vertical (Fig. $1 D$ ) and an islet cell (data not shown) were observed. However, other LT-driven GABAergic neurons did not resemble any of the known morphological classes previously described (Fig. 1E) (Heinke et al., 2004; Yasaka et al., 2007).

\section{$\mathrm{EGFP}^{+}$GABAergic neurons with convergent input (low and high} threshold) or only high-threshold input The combination of different threshold afferent fiber types that activate a given GABAergic neuron could provide insight into that neuron's role in sensory processing. For example, GABAergic neurons with $\mathrm{A} \beta$ fiber input could act in a feedforward manner to prevent nociceptive projection neurons from being activated by similar LT afferents. If such GABAergic neurons had additional input from HT fibers, as is the case with the neuron in Figure 2, this might indicate a role in the gating of pain or temperature information in addition to potentially suppressing LT drive.

Nearly all of the GABAergic neurons found to have $\mathrm{A} \beta$ fiber input also had convergent input from HT fibers. These could be distinguished from those neurons that received exclusively HT fiber drive $(65 \%$, 26/40) (Fig. 2E, gray-shaded plus horizontal-striped wedges), or no detectable input (15\% 6/40) (Fig. 2E, white wedge). A total of $37.5 \%$ of GABAergic neurons were activated by $\mathrm{A} \delta$ fibers (15/40) (Fig. 2E, horizontal-striped wedges) and $70 \%$ by C fibers (28/40, gray-shaded wedges), with some overlap between these subsets. This included 7.5\% (3/40) with monosynaptic input from A $\delta$ fibers, and 28\% (11/40) with monosynaptic C-fiber input (not illustrated). HT (A $\delta$ and/or C) synaptic drive, including both monosynaptic and polysynaptic, activated the majority of the GABAergic neurons tested $(\sim 83 \%$, 33/40).

\section{Firing patterns of neurons with convergent or high-threshold input}

Action potential firing patterns have been used to help identify subclasses of inhibitory neurons in vitro (Thomson et al., 1989; Ruscheweyh and Sandkühler, 2002; Lu and Perl, 2003). Furthermore, although firing patterns recorded in response to current steps are not predictors of the types of cutaneous stimuli that will activate a dorsal horn neuron or the exact response profile of the firing pattern evoked by natural stimuli in vivo, they can provide insight into important response features such as readiness to fire 
A TONIC

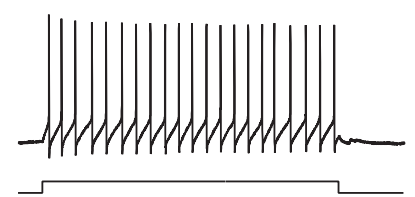

C SINGLE SPIKE

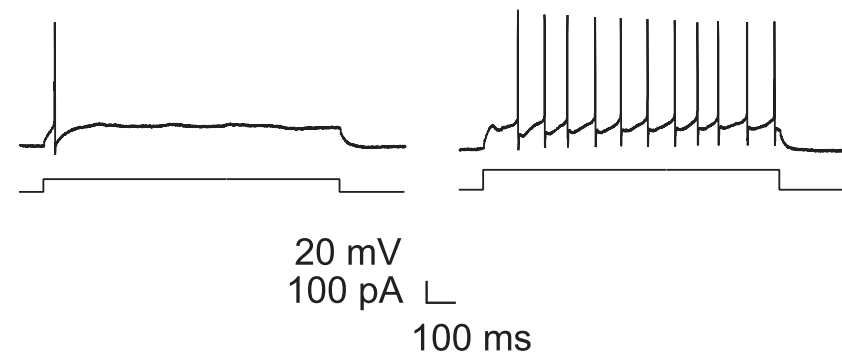

E

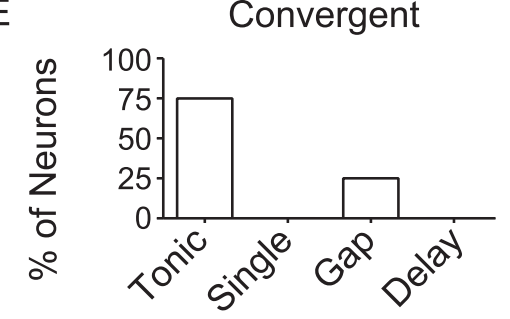

$\mathrm{F}$

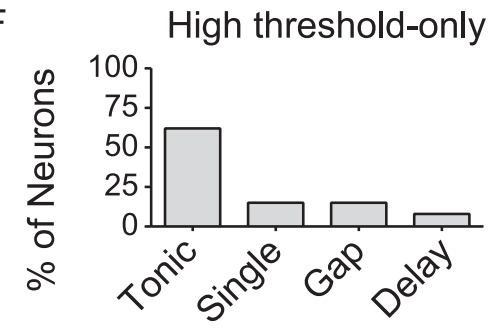

Figure 3. Comparison of firing patterns of GABAergic neurons with convergent input (low and high threshold) or only high-threshold input. Tonic $(\boldsymbol{A})$, gap $(\boldsymbol{B})$, single spike ( $\boldsymbol{C}$, and delay (D) firing patterns were recorded from different GABAergic neurons during $1 \mathrm{~s}$ depolarizing current steps. $\boldsymbol{E}, \boldsymbol{F}$, The proportion of each type of firing pattern among $(\boldsymbol{E})$ convergent $(n=4)$ versus $(\boldsymbol{F})$ high-threshold-only input GABAergic neurons $(n=13)$.

action potentials (Graham et al., 2007). Therefore, we wanted to determine the action potential firing patterns of the novel subset of GABAergic neurons defined here as receiving convergent LT and HT input and compare these to the group that only responded to HT input.

Action potentials were recorded in current clamp mode in response to one second long depolarizing current steps in a subset of the EGFP ${ }^{+}$neurons. Figure 3, $A-D$, shows examples of the firing patterns observed in $\mathrm{EGFP}^{+}$GABAergic neurons. Of the neurons with either convergent or HT-only input, 17/19 had firing patterns that could be classified based on previously defined criteria (Ruscheweyh and Sandkühler, 2002; Heinke et al., 2004). By far, the most common firing pattern among the convergent group was tonic firing, including $75 \%$ of the neurons tested (Fig. $3 A, E$ ). Gap-firing neurons were also found, but were more infrequent $(25 \%)$ (Fig. $3 B, E$ ). Figure $3 F$ shows the firing patterns found in the HT-only group of GABAergic neurons. Similar to the convergent group, tonic firing was the most common pattern in the HT-only group $(62 \%)$ and gap firing was more infrequent (15\%). However, there were 2 additional firing patterns encountered in this group and at rare occurrence: single spike (15\%) (Fig. 3C,F), and delay firing (8\%) (Fig. 3D,F). While the convergent and HT-only neurons may be distinguishable based on their afferent inputs, most of the GABAergic neurons from these two subclasses had similar firing patterns.

\section{Low-threshold drive onto $\mathrm{EGFP}^{+} \mathrm{GABAergic}$ neurons is more prevalent at younger ages}

Low-threshold fiber-driven pathways in the dorsal horn may undergo rearrangements during postnatal maturation (Fitzgerald, 1985; Jennings and Fitzgerald, 1998; Park et al., 1999; Nakatsuka et al., 2000; Torsney and Fitzgerald, 2002). Therefore, we wanted to address whether the prevalence of LT primary afferent synaptic drive onto $\mathrm{EGFP}^{+}$GABAergic neurons changes during maturation. However, we were uncertain whether $A \beta$ fibers have the same thresholds for activation in the developing versus mature dorsal root. Therefore, to avoid misclassification of $\mathrm{A} \beta$ fiber evoked synaptic responses at younger ages, we determined the stimulus intensity thresholds for $\mathrm{A} \beta$ fibers. Extracellular compound action potentials were recorded from 7, 5, and 8 L3-L5 dorsal roots taken from 3 neonatal (P7-P11), 3 juvenile (P17$\mathrm{P} 24)$ and 3 mature (P30-P45) mice, respectively. Figure $4 \mathrm{~A}$ shows a schematic of the dorsal root recording setup. Figure 4, $B-D$, shows compound action potentials recorded from the same P11 dorsal root at different stimulation intensities. Figure $4 B$ is an example of a typical tri-phasic compound action potential, which was the fastest detected $(1.53 \mathrm{~m} / \mathrm{s})$ and could be elicited with low intensity stimuli $(25 \mu \mathrm{A})$, consistent with $\mathrm{A} \alpha$ and $\mathrm{A} \beta$ fibers (arrow). Additional slower components of the compound action potentials were recruited with higher stimulation intensities, consistent with $\mathrm{A} \delta$ (Fig. $4 C$, filled arrowhead) and C (Fig. $4 D$, open arrowhead) fibers. The mean conduction velocities $( \pm \mathrm{SE})$ for the $\mathrm{A} \alpha / \mathrm{A} \beta$ components were $0.8 \pm 0.2,1.1 \pm 0.1$, and $1.6 \pm 0.3 \mathrm{~m} / \mathrm{s}$ for neonatal, juvenile and mature roots, respectively. These were not significantly different among age groups $(p>0.05)$.

Normalized stimulus-response relationships for the $\mathrm{A} \alpha / \mathrm{A} \beta$ components of the compound action potential were pooled for each age group and plotted for comparison in Figure $4 E$. There is a slight shift in the minimal intensity required to activate $\mathrm{A} \alpha / \mathrm{A} \beta$ fibers in neonates versus older ages. However, the stimulation intensity ranges remain similar across all ages. Furthermore, the maximal intensity we used to activate all $\mathrm{A} \alpha / \mathrm{A} \beta$ fibers in the mature dorsal root $(25 \mu \mathrm{A})$ was adequate even for neonatal dorsal roots, where higher intensity thresholds might be expected. Therefore, we conclude that our stimulation criteria for activating $\mathrm{A} \beta$ fibers at mature ages are also appropriate for younger ages.

To address whether the prevalence of LT drive onto GABAergic neurons changes during maturation, additional synaptic recordings were made from 41 neonatal (P7-P11) and 38 juvenile (P17-P24) $\mathrm{EGFP}^{+}$GABAergic neurons. We determined whether the minimal stimulation intensity required to evoke synaptic $A \beta$ fiber responses in $\mathrm{EGFP}^{+}$GABAergic neurons changed as a function of maturation. Mean ( $\pm \mathrm{SE}$ ) current values found to minimally stimulate synaptic activity were similar: $12.5 \pm 1.0,15.4 \pm$ 1.3 , and $11.5 \pm 1.4 \mu \mathrm{A}$ for neonatal $(n=17)$, juvenile $(n=16)$, and mature $(n=8)$ GABAergic neurons, respectively $(p>0.05)$. 
A

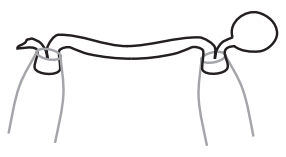

B $25 \mu \mathrm{A}$

C $100 \mu \mathrm{A}$

D $300 \mu \mathrm{A}$
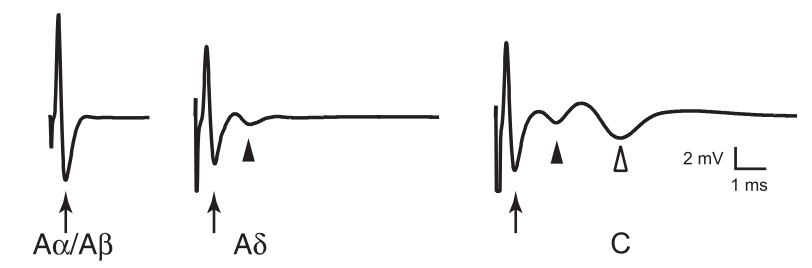

$\mathrm{E}$

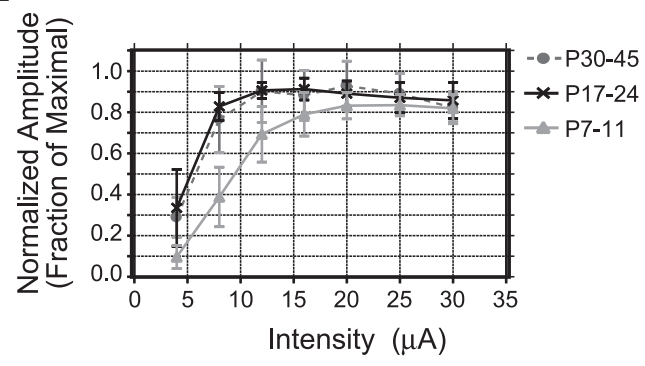

Figure 4. Similar stimulus threshold intensities for $A \beta$ fibers were observed at all ages. $A$, Schematic of the experimental setup to record compound action potentials from L3-L5 dorsal roots is shown. A suction electrode was placed at one end to stimulate and another at the opposite end to record extracellular compound action potentials. $\boldsymbol{B}-\boldsymbol{D}$, Representative compound action potentials recorded from the same $\mathrm{P} 11$ dorsal root at different stimulation intensities. $B$, An example of an $A \alpha / A \beta$ fiber component (arrow), which had the lowest threshold and fastest conduction velocity $(1.53 \mathrm{~m} / \mathrm{s})$. $C$, The $A \delta$ component (filled arrowhead) had a higher threshold and a slower conduction velocity $(0.42 \mathrm{~m} / \mathrm{s})$. D, The C-fiber component (open arrowhead) had the highest threshold and the slowest conduction velocity $(0.19 \mathrm{~m} / \mathrm{s})$. Each trace is an average of five consecutive sweeps at low frequency $(0.2 \mathrm{~Hz})$. $\boldsymbol{E}$, Similar stimulus response relationships were observed for the $A \alpha / A \beta$ fiber component of the compound action potential at different ages (P7-P11, P17-P24 and P30-P45). The A $\alpha / A \beta$ fiber amplitude recorded at each intensity was normalized to the maximal response (fraction of maximal) for each root, and then these plots were averaged among each age group.

The minimal stimulation ranges were $8-20,8-25$ and $4-16 \mu \mathrm{A}$ for neonatal, juvenile, and mature neurons, respectively.

Forty-two, 42 , and $20 \%$ of the lamina I and II GABAergic neurons responded to stimulation of LT fibers in the neonatal, juvenile and mature dorsal horn, respectively (Fig. $5 A$, gray bars). The prevalence of low-threshold input changed significantly as a function of age $(p<0.05)$. Low-threshold input was predominantly polysynaptic at all postnatal ages with monosynaptic $A \beta$ fiber input only observed rarely, but to a similar extent in neonatal, juvenile and mature mice (Fig. $5 A$, black bars) ( $p>0.05$, compared across ages). Therefore, we conclude that the proportion of GABAergic neurons driven by LT afferents decreases after the third postnatal week, but there is still a large number (20\%) of mature GABAergic neurons with LT fiber input at the oldest ages tested.

$\mathrm{A} \beta$ and $\mathrm{A} \delta$ fiber thresholds can overlap at stimulation intensity values between 20 and $25 \mu \mathrm{A}$ in mouse (our unpublished observation), as well as rat (Nakatsuka et al., 2000). To be certain that our identification of GABAergic neurons receiving LT synaptic drive was reliable, we assessed the occurrence of LT drive after imposing stricter, nonoverlapping criteria. When we only considered $4-16 \mu \mathrm{A}$ to be $\mathrm{A} \beta$ stimulation range, we found $\mathrm{A} \beta$ fiber input prevalence to be 37,30 and $20 \%$ in neonatal, juvenile, and mature neurons, respectively.

While the percentage of GABAergic neurons receiving LT synap- tic drive tends to decrease as developmental maturation proceeds, this does not necessarily mean that synaptic strength is diminished. It is synaptic strength that will determine the output of each GABAergic neuron under LT influence. Therefore, we determined whether the strength of LT synaptic drive onto GABAergic neurons changes with age. Peak amplitudes of evoked responses were measured, averaged and compared (Fig. $5 B-E$ ). Example traces of LT responses stimulated at $25 \mu \mathrm{A}$ from neonatal, juvenile, and mature GABAergic neurons are shown in Figure 5, $B-D$. The mean peak amplitude did not change significantly between neonatal, juvenile, and mature time points, respectively (Fig. $5 E)(p>0.05)$ although there is intrinsic variability of the peak amplitudes among neurons.

The majority of responses evoked by $\mathrm{A} \beta$ fiber stimulation appeared as complex polysynaptic waveforms with multiple peaks. Therefore, to assess whether the magnitude of this LT drive was changing over the course of postnatal maturation, we also compared the area under the curve (integrated current) of the responses evoked at $25 \mu \mathrm{A}$ among the three age groups. Figure $5 F$ shows the mean integrated synaptic current for $A \beta$ fiber evoked responses at all ages tested. There were no significant differences in the integrated current across age groups $(p>0.05)$. However, there is a trend toward decreasing variability of response magnitudes that is accompanied by a trend of decreased mean integrated currents as the animals mature.

Receptive field sizes and cutaneous thresholds for mechanical stimulation of dorsal horn neurons change substantially over the first 3 weeks of postnatal development (Fitzgerald, 1985; Torsney and Fitzgerald, 2002). Recent studies have suggested that these changes may be due to changes in inhibitory control at the network level (Bremner and Fitzgerald, 2008). We determined whether the two groups of GABAergic neurons characterized as convergent or HT-only in the P30-P40 mice were also present in younger mice with the same prevalence. Figure 6 diagrams the proportion of EGFP $^{+}$GABAergic neurons receiving different classes of afferent input at different developmental time points. For each pie chart in Figure 6, black wedges represent the percentage of neurons that had input exclusively from HT fibers (HT-only). These are distinct from neurons that received inputs from both low and HT fibers (convergent input, dark gray wedges). A small subset of GABAergic neurons had input only from $\mathrm{A} \beta$ fibers (light gray wedges), and for a few neurons, no input could be detected (white wedges). Although there was a trend toward an increase in the prevalence of HT-only input, this was not significantly different across ages $(p>0.05)$. In contrast, there was a significant change in prevalence of convergent input with age $(p<0.05)$. However at all ages, including the oldest group, neurons with convergent input constitute a major subclass of $\mathrm{EGFP}^{+}$GABAergic neurons.

The magnitude of the $\mathrm{A} \beta$ fiber drive onto the convergent input neurons did not change during maturation (Fig. 5). However, HT input was a common feature to both convergent input interneurons as well as HT-only interneurons. Therefore, we wanted to determine whether there were developmental differences in the magnitude of this HT input between the two major subclasses of interneuron. If so, it could reflect differences in the specific subtypes of HT afferents that drive these different GABAergic neuron subclasses and would further distinguish them. Figure 7A shows the magnitude of the HT input onto convergent-input neurons. Although there is intrinsic variability, especially in the younger age groups, and the trend is a decrease in synaptic strength, the mean integrated current was not significantly different among age groups $(p>0.05)$. In contrast, in HT-only neurons, the magnitude of the integrated current of the maximal response decreased significantly between neonatal and juvenile ages as well as between neonatal and mature ages $\left({ }^{*} p<0.05\right)$. 
The decreasing synaptic strength of the HT drive during postnatal maturation is interesting, because it contrasts with previous reports showing C-fiber inputs increasing in strength during development (Fitzgerald, 1985, 1988; Jennings and Fitzgerald, 1998). However, those studies were done at an earlier age range than our study, making it difficult to directly compare. Nevertheless, our data imply that there is postnatal remodeling in the synaptic network activated by HT fibers. Overall, maturation of the HT afferent drive onto convergent-input neurons differs from that of the HT-only group. These differences support our conclusion that these are 2 functionally distinct subclasses of GABAergic neurons.

\section{Discussion}

We have identified a novel subclass of GABAergic neurons in lamina I and II of the dorsal horn that receive convergent LT and HT afferent drive. Although the prevalence of this LT drive decreases after the third week, a significant proportion remains, suggesting that these neurons are a feature of the adult SDH. The convergent nature of the input to these neurons suggests that they play a role in gating innocuous as well as nociceptive sensory input, and could be involved in preventing the pathological flow of LT information to nociceptive output neurons.

\section{Low-threshold afferent drive onto GABAergic neurons}

Most incoming nociceptive and thermoreceptive signals carried by $\mathrm{HT} \mathrm{A} \delta$ and C-fiber afferents terminate in the SDH (Light and Perl, 1979), where there are many inhibitory neurons (Todd and Sullivan, 1990) that can modify those incoming signals. Although recent studies have defined LT A $\beta$ afferent drive onto lamina III inhibitory neurons (Schneider and Lopez, 2002), thus far only HT drive has been documented for lamina II inhibitory neurons ( $\mathrm{Lu}$ and Perl, 2003; Heinke et al., 2004; Hantman et al., 2004), including $\mathrm{EGFP}^{+}$GABAergic neurons from the same transgenic mouse as in our experiments (Heinke et al., 2004). Interestingly, however, inhibitory currents could be evoked by LT A $\delta$ fiber stimulation in the SDH (Yoshimura and Nishi, 1995; Narikawa et al., 2000). In addition, inhibitory islet cells in lamina II have lower threshold C-fiber inputs, consistent with LT mechanoreceptors or cool thermoreceptors (Lu and Perl, 2003). However, none of these studies report LT A $\beta$ fiber activation of inhibitory SDH neurons. This may be due to differences in slice parameters, neurons sampled, or stimulation intensities tested.

The LT drive studied here is most likely carried by A $\beta$ sensory neurons because the thresholds for initial responses were below the A $\delta$ fiber range. Nevertheless, we cannot exclude the possibility that additional innocuous cutaneous information is carried by $\mathrm{D}$ hair

$E$
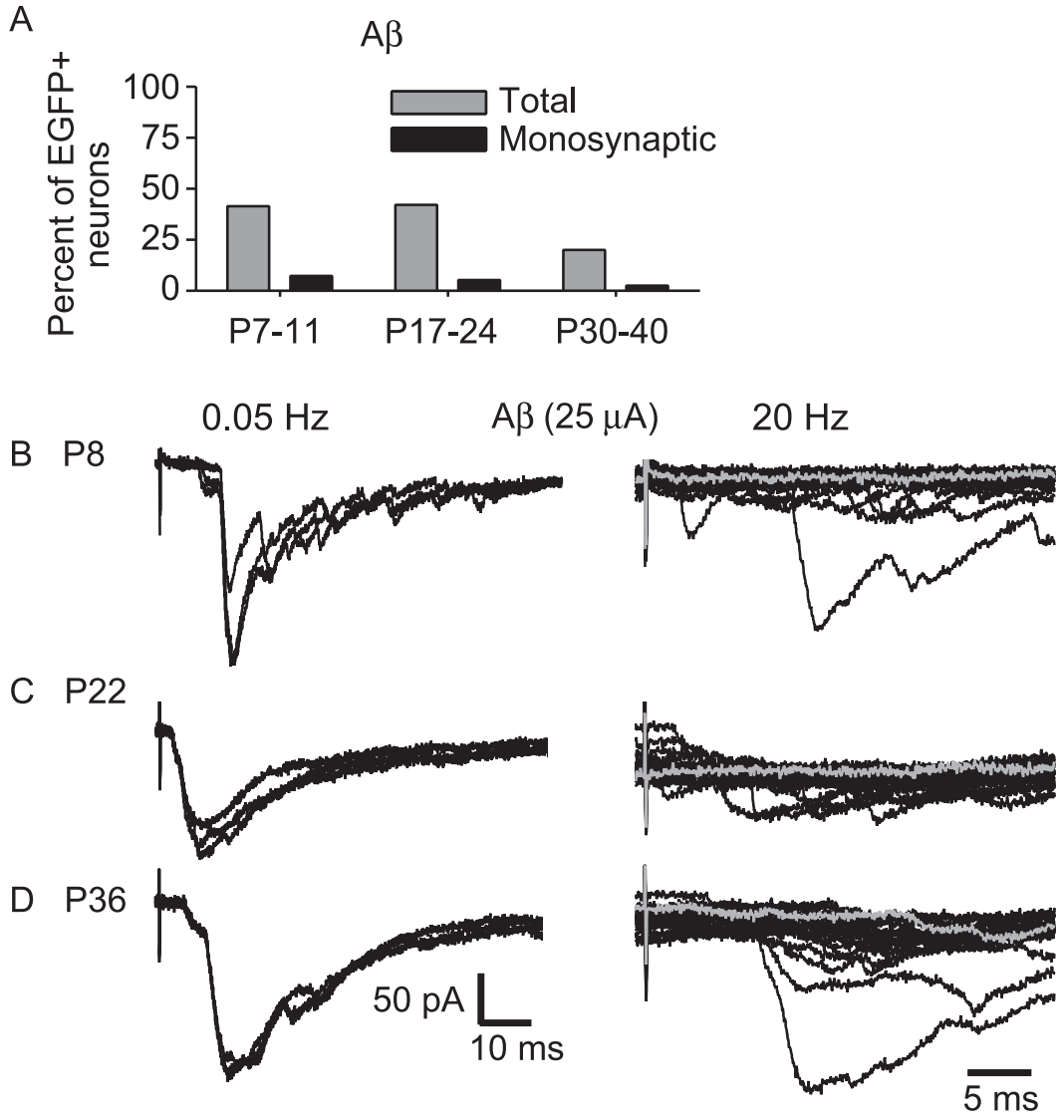

$\mathrm{F}$
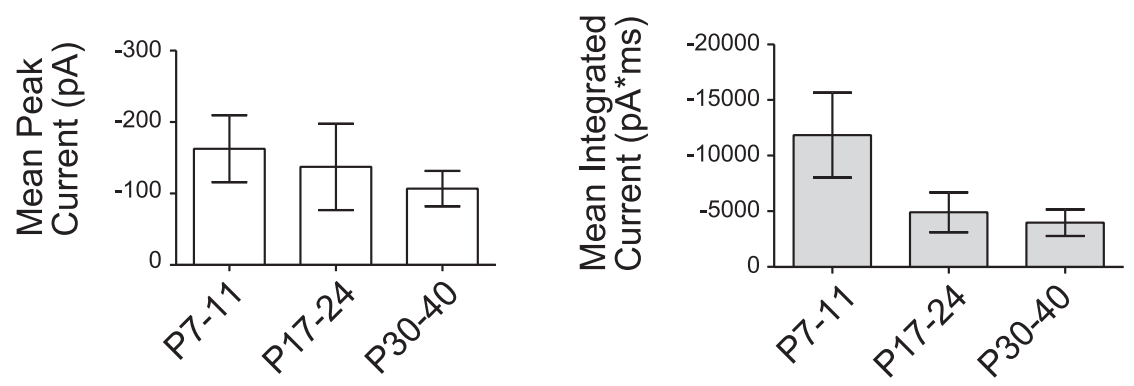

Figure 5. The prevalence of $A \beta$ fiber drive onto EGFP-labeled GABAergic neurons decreased beyond the third postnatal week, but the strength of $A \beta$ fiber drive was similar during maturation. $A$, The prevalence of $A \beta$ fiber input to $G A B A$ ergic neurons at different ages is shown. "Total" includes inputs that are monosynaptic or polysynaptic (gray bars). The prevalence of monosynaptic $A \beta$ fiber input only is also shown (black bars). $\boldsymbol{B}-\boldsymbol{D}$, Examples of $A \beta$ fiber input in response to $25 \mu A$ stimulation recorded from neonatal (P8) (B), juvenile (P22) ( $(\boldsymbol{C}$, and mature (P36) (D) GABAergic neurons. On the left are three consecutive traces at low-frequency stimulation $(0.05 \mathrm{~Hz})$, and on the right, 20 consecutive responses recorded at high frequency $(20 \mathrm{~Hz}$, shown at an expanded timescale). In all three cases $(\boldsymbol{B}-\boldsymbol{D})$, there are failures at high frequency, consistent with a polysynaptic input (illustrated by gray traces). $\boldsymbol{E}$, The average peak amplitude of $A \beta$ fiber evoked responses is compared across age groups (17 neonatal, 16 juvenile, and 8 mature neurons; $p>0.05$, one-way ANOVA, Newman-Keuls multiple comparison test). $\boldsymbol{F}$, The average magnitude of $A \beta$ fiber input was measured as mean of the integrated current for responses to $25 \mu \mathrm{A}$ stimuli at low frequency and compared across all age groups (17 neonatal, 16 juvenile, and 7 mature neurons; $p>0.05$, one-way ANOVA, Newman-Keuls multiple comparison test). $\boldsymbol{E}, \boldsymbol{F}$, Error bars represent SEM.

(A $\delta$ ) fibers or LT mechanosensitive C fibers (Burgess and Perl, 1967; Lynn and Carpenter, 1982; Sugiura et al., 1986; Traub and Mendell, 1988; Ritter and Mendell, 1992; Koltzenburg et al., 1997; Woodbury et al., 2001; Lu and Perl, 2003; Boada and Woodbury, 2008). However, we probably did not misclassify these as $\mathrm{A} \beta$ fibers, because there is a distinction between thresholds for cutaneous versus electrical stimuli. D hair A $\delta$ fibers or LT mechanosensitive $\mathrm{C}$ fibers should have higher electrical thresholds than $\mathrm{A} \beta$ fibers $(\geq 25 \mu \mathrm{A})$, due to smaller axonal diameter and relative lack of myelination. In 

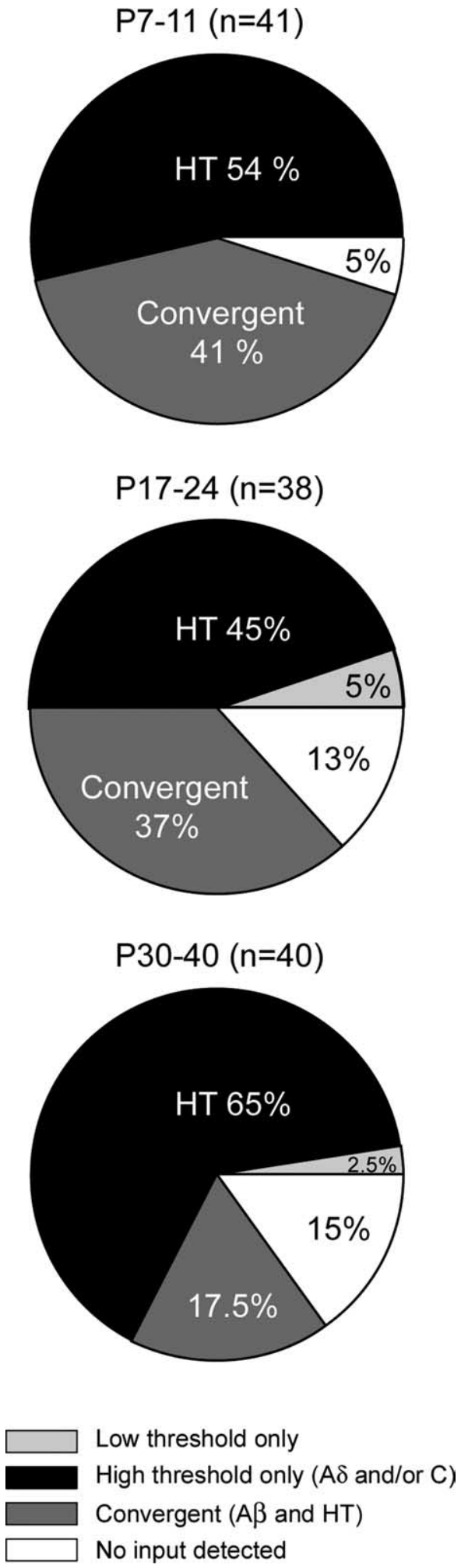

Figure 6. A summary of the distribution of afferent inputs onto GABAergic neurons compared across different ages. The majority of GABAergic neurons fell into two categories based on afferent input: neurons with only high-threshold ( $A \delta$ and/or C) fiber input or those with convergent input from low-threshold $(A \beta)$ and high-threshold fibers. The wedges in the pie charts represent the proportion of neurons that received only low-threshold fiber input (light gray), only high-threshold input (black), both low and high-threshold input ("convergent", dark gray), or no detectable input (white). The most common groups (black and dark gray) were observed at a similar frequency during the first 3 weeks (P7-P24), but there was a reduction in the prevalence of convergent neurons at older ages ( $p<0.05, \chi^{2}$ test for trend).
A

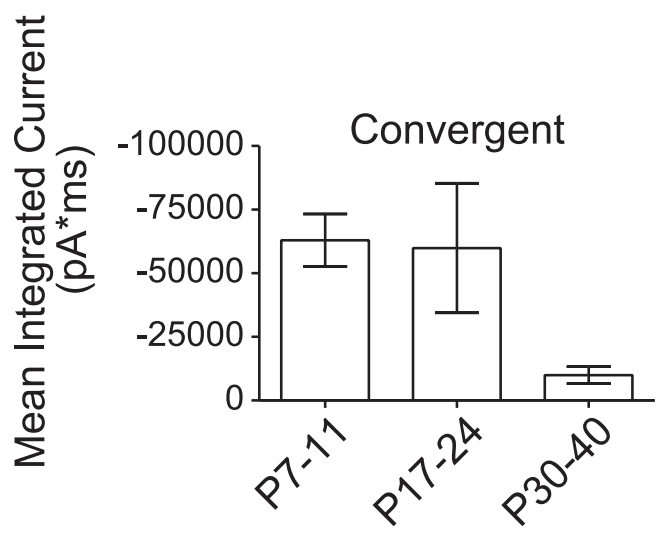

B

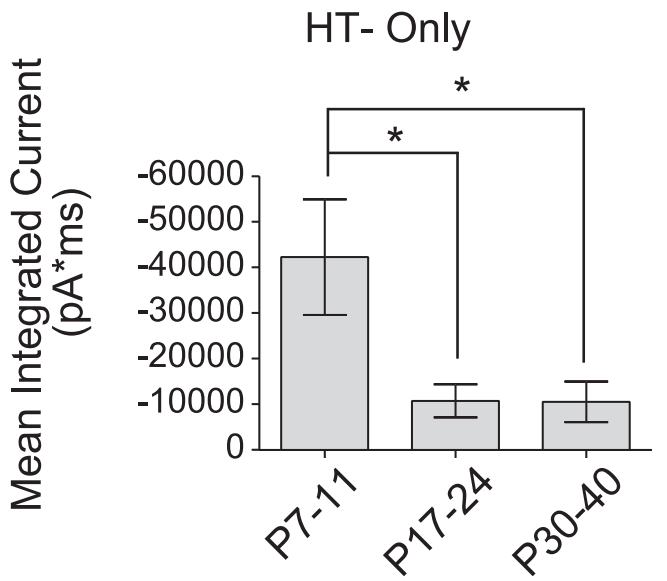

Figure 7. Changes in the magnitude of high-threshold responses during maturation. The mean integrated current for responses evoked by maximal intensity ( 300 or $500 \mu \mathrm{A}$, at low frequency) is compared across different ages in the two major categories of neurons: ( $\boldsymbol{A})$ those with convergent input, and $(\boldsymbol{B})$ those with input only from high-threshold fibers. $\boldsymbol{A}$, Mean integrated current was calculated by subtracting the integrated synaptic current evoked at 25 $\mu \mathrm{A}$ from that recorded at the maximal stimulation intensity. For convergent-input neurons, there was no significant difference in the mean integrated current in response to high-threshold input across age groups ( 17 neonatal, 12 juvenile, and 6 mature neurons; $p>0.05$, one-way ANOVA, Newman-Keuls multiple comparison test). $\boldsymbol{B}$, For the high-threshold-only neurons, there was a significant difference in mean integrated current between neonatal and juvenile ages, as well as between neonatal and mature ages ( 22 neonatal, 17 juvenile, and 26 mature neurons; ${ }^{*} p<0.05$, one-way ANOVA, Newman-Keuls multiple comparison test). Error bars represent SEM.

convergent neurons, responses to these afferents would overlap with $\mathrm{HT}$ responses, contrary to our A $\beta$ fiber inputs. Afferents with low mechanical, but high electrical thresholds may nevertheless contribute to the excitatory pathway producing allodynia in the disinhibited dorsal horn. In addition, some of our $A \beta$ fiber input may be from nociceptors (Koerber et al., 1988; Ritter and Mendell, 1992; Djouhri et al., 1998; Djouhri and Lawson, 2004; Boada and Woodbury, 2008 ), although the majority of $A \beta$ fibers selectively carry innocuous touch sensation (Djouhri and Lawson, 2004).

\section{High-threshold afferent drive}

Why do most of the GABAergic neurons with LT drive also have convergent HT drive? The balance of excitation and inhibition is important for both development and adult CNS function (Akerman and Cline, 2007). Therefore, these neurons could be important in gating or modulating noxious as well as innocuous infor- 
mation, implying that their targets could also receive similar synaptically mediated information. Some projection neurons are excited by both HT and LT fibers with many more showing novel LT input accompanying disinhibition (Torsney and MacDermott, 2006). Convergent-input GABAergic neurons would be perfectly suited to counter balance that excitatory drive.

\section{Action potential firing patterns as an identifying characteristic of cell type}

Action potential firing patterns in response to depolarizing current steps have been used along with other criteria to classify dorsal horn neurons (Han et al., 1998; Grudt and Perl, 2002). The convergentinput and HT-only subclasses of GABAergic neurons have similar firing patterns, with tonic firing being dominant for both. This higher prevalence of tonic firing $\mathrm{EGFP}^{+}$neurons than in previous reports (Heinke et al., 2004; Dougherty et al., 2005) is surprising. However, islet cells were shown to fire tonically (Grudt and Perl, 2002; Lu and Perl, 2003), and $\sim 60 \%$ of lamina II EGFP ${ }^{+}$GABAergic neurons are islet cells (Heinke et al., 2004). This discrepancy could be due to a difference in neurons sampled. Interestingly, Dougherty and others (2005) found lamina I GABAergic neurons that could switch between tonic and initial burst patterns, possibly due to modulation of membrane properties by endogenous modulators, such as monoamines (Garraway and Hochman, 2001).

\section{Maturation of low-threshold drive}

Whether synaptic activation of SDH neurons by $\mathrm{A} \beta$ fibers changes during postnatal maturation has been a matter of some debate. The majority of LT synaptic input we report is polysynaptic, with potential involvement of more ventral excitatory interneurons. Furthermore, the rare monosynaptic LT input could be onto ventrally extending dendrites (Fig. 1D). Therefore, our data do not have a direct bearing on the laminar location of $\mathrm{A} \beta$ fiber terminals.

Consistent with our data, physiologically measured monosynaptic and polysynaptic $\mathrm{A} \beta$ fiber synaptic drive onto unidentified lamina II neurons was shown to extend beyond the third postnatal week, but diminishes at older ages when tested in vitro (Park et al., 1999; Nakatsuka et al., 2000). However, these changes were more dramatic than we observe (only $\sim 8-9 \%$ remain, compared with our $20 \%$ ). In vivo extracellular recordings show a reduction in neurons responding to LT fiber stimulation, or innocuous touch during the first 3 postnatal weeks (Fitzgerald and Jennings, 1999; Torsney and Fitzgerald, 2002). These observations in vivo contrast with our stable LT drive over this time, possibly because some of our responses are subthreshold for action potentials.

Inhibitory neurons account for only $\sim 30 \%$ of the SDH population and our study focuses specifically on a subset of those. In previous studies, neurons were sampled randomly causing a probable bias toward excitatory neurons, given that those represent the majority of SDH neurons. Therefore, previous data could reflect greater LT-driven excitation at younger ages. Together with our data, LT-driven excitation would be balanced by LT-driven inhibition at the youngest ages. However, in neonates the weaker chloride extrusion capacity of immature SDH neurons (Cordero-Erausquin et al., 2005) could lead to depolarizing GABA and glycine responses and to predominantly excitatory LT drive, consistent with greater sensitivity to innocuous touch seen at younger ages in the SDH (Fitzgerald, 1985; Jennings and Fitzgerald, 1998; Torsney and Fitzgerald, 2002).

\section{Functional significance of LT-driven inhibition}

Pharmacological disruption of inhibition within the rat dorsal horn in vivo results in allodynia (Yaksh, 1989), commonly a fea- ture of chronic pain. This effect is dose-dependent and specific to the rostrocaudal site of the antagonists, suggesting that a LT excitatory pathway onto nociceptive neurons is present in the dorsal horn, but is normally silenced by strong inhibitory control. Further support for this hypothesis comes from in vitro studies, where only intrinsic spinal inhibition is intact (Baba et al., 2003; Torsney and MacDermott, 2006). Disinhibition reveals novel polysynaptic $A \beta$ fiber drive onto projection neurons in lamina I bearing the NK1 receptor $\left(\mathrm{NK}_{1} \mathrm{R}^{+}\right.$) (Torsney and MacDermott, 2006; Keller et al., 2007). This further implicates LT-driven pathways in chronic pain states, because intact lamina I NK1R ${ }^{+}$neurons are required for the development of chronic pain (Nichols et al., 1999). PKC $\gamma$ signaling is also implicated in this pathway (Malmberg et al., 1997; Miraucourt et al., 2007), and occurs in a subset of putative excitatory neurons in inner lamina II (Polgar et al., 1999) that receive innocuous inputs (Neumann et al., 2008).

Because LT pathways are normally silenced by local inhibition, intact inhibition in the dorsal horn is essential for normal sensory processing. Although it remains to be shown how tonic inhibition may contribute to this suppression, we identified LTdriven GABAergic neurons that are located in the SDH, making them good candidates to suppress polysynaptic LT activation of projection neurons. Such feedforward inhibition is common to the CNS (Buzsáki, 1984; Miller et al., 2001; Nakatsuka et al., 2005) and could be a powerful means of gating inappropriate sensory signals in the pain pathway.

Finally, it is interesting that GABAergic neurons receiving LT drive in the dorsal horn, such as those reported here, are predicted by the gate control theory of Melzack and Wall (1965). Innocuous cutaneous stimuli can strongly influence the processing of acute pain, as when rubbing a sore area reduces discomfort. To explain this, the gate control theory proposes that nociceptive projection neurons are activated by noxious afferent input, which "opens" the gate, while LT, non-noxious fibers inhibit this signal and "close" gate (Melzack and Wall, 1965). This would predict a flow of LT drive onto inhibitory neurons, which would then prevent $\mathrm{HT}$ activation of projection neurons. The LT-driven GABAergic neurons described here could be an element of that proposed circuit.

\section{References}

Akerman CJ, Cline HT (2007) Refining the roles of GABAergic signaling during neural circuit formation. Trends Neurosci 30:382-389.

Baba H, Ji RR, Kohno T, Moore KA, Ataka T, Wakai A, Okamoto M, Woolf CJ (2003) Removal of GABAergic inhibition facilitates polysynaptic A fibermediated excitatory transmission to the superficial spinal dorsal horn. Mol Cell Neurosci 24:818-830.

Baccei ML, Fitzgerald M (2004) Development of GABAergic and glycinergic transmission in the neonatal rat dorsal horn. J Neurosci 24:4749-4757.

Beyer C, Roberts LA, Komisaruk BR (1985) Hyperalgesia induced by altered glycinergic activity at the spinal cord. Life Sci 37:875-882.

Bicknell HR Jr, Beal JA (1984) Axonal and dendritic development of substantia gelatinosa neurons in the lumbosacral spinal cord of the rat. J Comp Neurol 226:508-522.

Boada MD, Woodbury CJ (2008) Myelinated skin sensory neurons project extensively throughout adult mouse substantia gelatinosa. J Neurosci 28:2006-2014.

Bremner LR, Fitzgerald M (2008) Postnatal tuning of cutaneous inhibitory receptive fields in the rat. J Physiol 586:1529-1537.

Burgess PR, Perl ER (1967) Myelinated afferent fibres responding specifically to noxious stimulation of the skin. J Physiol 190:541-562.

Buzsáki G (1984) Feed-forward inhibition in the hippocampal formation. Prog Neurobiol 22:131-153.

Cordero-Erausquin M, Coull JA, Boudreau D, Rolland M, De Koninck Y (2005) Differential maturation of GABA action and anion reversal potential in spinal lamina I neurons: impact of chloride extrusion capacity. J Neurosci 25:9613-9623. 
Djouhri L, Lawson SN (2004) Abeta-fiber nociceptive primary afferent neurons: a review of incidence and properties in relation to other afferent A-fiber neurons in mammals. Brain Res Brain Res Rev 46:131-145.

Djouhri L, Bleazard L, Lawson SN (1998) Association of somatic action potential shape with sensory receptive properties in guinea-pig dorsal root ganglion neurones. J Physiol 513:857-872.

Dougherty KJ, Sawchuk MA, Hochman S (2005) Properties of mouse spinal lamina I GABAergic interneurons. J Neurophysiol 94:3221-3227.

Erlander MG, Tillakaratne NJ, Feldblum S, Patel N, Tobin AJ (1991) Two genes encode distinct glutamate decarboxylases. Neuron 7:91-100.

Fitzgerald M (1985) The post-natal development of cutaneous afferent fibre input and receptive field organization in the rat dorsal horn. J Physiol 364:1-18.

Fitzgerald M (1988) The development of activity evoked by fine diameter cutaneous fibres in the spinal cord of the newborn rat. Neurosci Lett 86:161-166.

Fitzgerald M (2005) The development of nociceptive circuits. Nat Rev Neurosci 6:507-520.

Fitzgerald M, Jennings E (1999) The postnatal development of spinal sensory processing. Proc Natl Acad Sci U S A 96:7719-7722.

Fitzgerald M, Butcher T, Shortland P (1994) Developmental changes in the laminar termination of A fibre cutaneous sensory afferents in the rat spinal cord dorsal horn. J Comp Neurol 348:225-233.

Garraway SM, Hochman S (2001) Modulatory actions of serotonin, norepinephrine, dopamine, and acetylcholine in spinal cord deep dorsal horn neurons. J Neurophysiol 86:2183-2194.

Graham BA, Brichta AM, Callister RJ (2007) Pinch-current injection defines two discharge profiles in mouse superficial dorsal horn neurones, in vitro. J Physiol 578:787-798.

Grudt TJ, Perl ER (2002) Correlations between neuronal morphology and electrophysiological features in the rodent superficial dorsal horn. J Physiol 540:189-207.

Han ZS, Zhang ET, Craig AD (1998) Nociceptive and thermoreceptive lamina I neurons are anatomically distinct. Nat Neurosci 1:218-225.

Hantman AW, van den Pol AN, Perl ER (2004) Morphological and physiological features of a set of spinal substantia gelatinosa neurons defined by green fluorescent protein expression. J Neurosci 24:836-842.

Heinke B, Ruscheweyh R, Forsthuber L, Wunderbaldinger G, Sandkühler J (2004) Physiological, neurochemical and morphological properties of a subgroup of GABAergic spinal lamina II neurones identified by expression of green fluorescent protein in mice. J Physiol 560:249-266. Epub 2004 Jul 2029.

Jennings E, Fitzgerald M (1998) Postnatal changes in responses of rat dorsal horn cells to afferent stimulation: a fibre-induced sensitization. J Physiol 509:859-868.

Kaufman DL, McGinnis JF, Krieger NR, Tobin AJ (1986) Brain glutamate decarboxylase cloned in lambda gt-11: fusion protein produces gammaaminobutyric acid. Science 232:1138-1140.

Keller AF, Beggs S, Salter MW, De Koninck Y (2007) Transformation of the output of spinal lamina I neurons after nerve injury and microglia stimulation underlying neuropathic pain. Mol Pain 3:27.

Koerber HR, Druzinsky RE, Mendell LM (1988) Properties of somata of spinal dorsal root ganglion cells differ according to peripheral receptor innervated. J Neurophysiol 60:1584-1596.

Koltzenburg M, Stucky CL, Lewin GR (1997) Receptive properties of mouse sensory neurons innervating hairy skin. J Neurophysiol 78:1841-1850.

Light AR, Perl ER (1979) Spinal termination of functionally identified primary afferent neurons with slowly conducting myelinated fibers. J Comp Neurol 186:133-150.

Lu Y, Perl ER (2003) A specific inhibitory pathway between substantia gelatinosa neurons receiving direct C-fiber input. J Neurosci 23:8752-8758.

Lynn B, Carpenter SE (1982) Primary afferent units from the hairy skin of the rat hind limb. Brain Res 238:29-43.

Malmberg AB, Chen C, Tonegawa S, Basbaum AI (1997) Preserved acute pain and reduced neuropathic pain in mice lacking PKCgamma. Science 278:279-283.

Melzack R, Wall PD (1965) Pain mechanisms: a new theory. Science 150:971-979.

Miller KD, Pinto DJ, Simons DJ (2001) Processing in layer 4 of the neocortical circuit: new insights from visual and somatosensory cortex. Curr Opin Neurobiol 11:488-497.

Miraucourt LS, Dallel R, Voisin DL (2007) Glycine inhibitory dysfunction turns touch into pain through PKCgamma interneurons. PLoS ONE 2:e1116.
Nakatsuka T, Ataka T, Kumamoto E, Tamaki T, Yoshimura M (2000) Alteration in synaptic inputs through C-afferent fibers to substantia gelatinosa neurons of the rat spinal dorsal horn during postnatal development. Neuroscience 99:549-556.

Nakatsuka T, Chen M, Takeda D, King C, Ling J, Xing H, Ataka T, Vierck C, Yezierski R, Gu JG (2005) Substance P-driven feed-forward inhibitory activity in the mammalian spinal cord. Mol Pain 1:20.

Narikawa K, Furue H, Kumamoto E, Yoshimura M (2000) In vivo patchclamp analysis of IPSCs evoked in rat substantia gelatinosa neurons by cutaneous mechanical stimulation. J Neurophysiol 84:2171-2174.

Neumann S, Braz JM, Skinner K, Llewellyn-Smith IJ, Basbaum AI (2008) Innocuous, not noxious, input activates PKCgamma interneurons of the spinal dorsal horn via myelinated afferent fibers. J Neurosci 28:7936-7944.

Nichols ML, Allen BJ, Rogers SD, Ghilardi JR, Honore P, Luger NM, Finke MP, Li J, Lappi DA, Simone DA, Mantyh PW (1999) Transmission of chronic nociception by spinal neurons expressing the substance $\mathrm{P}$ receptor. Science 286:1558-1561.

Oliva AA Jr, Jiang M, Lam T, Smith KL, Swann JW (2000) Novel hippocampal interneuronal subtypes identified using transgenic mice that express green fluorescent protein in GABAergic interneurons. J Neurosci 20:3354-3368.

Park JS, Nakatsuka T, Nagata K, Higashi H, Yoshimura M (1999) Reorganization of the primary afferent termination in the rat spinal dorsal horn during post-natal development. Brain Res Dev Brain Res 113:29-36.

Polgár E, Fowler JH, McGill MM, Todd AJ (1999) The types of neuron which contain protein kinase $\mathrm{C}$ gamma in rat spinal cord. Brain Res 833:71-80.

Ritter AM, Mendell LM (1992) Somal membrane properties of physiologically identified sensory neurons in the rat: effects of nerve growth factor. J Neurophysiol 68:2033-2041.

Roberts LA, Beyer C, Komisaruk BR (1986) Nociceptive responses to altered GABAergic activity at the spinal cord. Life Sci 39:1667-1674.

Ruscheweyh R, Sandkühler J (2002) Lamina-specific membrane and discharge properties of rat spinal dorsal horn neurones in vitro. J Physiol 541:231-244.

Schneider SP, Lopez M (2002) Immunocytochemical localization of glutamic acid decarboxylase in physiologically identified interneurons of hamster spinal laminae III-V. Neuroscience 115:627-636.

Sugiura Y, Lee CL, Perl ER (1986) Central projections of identified, unmyelinated (C) afferent fibers innervating mammalian skin. Science 234:358-361.

Thomson AM, West DC, Headley PM (1989) Membrane Characteristics and synaptic responsiveness of superficial dorsal horn neurons in a slice preparation of adult rat spinal cord. Eur J Neurosci 1:479-488.

Todd AJ, Sullivan AC (1990) Light microscope study of the coexistence of GABA-like and glycine-like immunoreactivities in the spinal cord of the rat. J Comp Neurol 296:496-505.

Torsney C, Fitzgerald M (2002) Age-dependent effects of peripheral inflammation on the electrophysiological properties of neonatal rat dorsal horn neurons. J Neurophysiol 87:1311-1317.

Torsney C, MacDermott AB (2006) Disinhibition opens the gate to pathological pain signaling in superficial neurokinin 1 receptor-expressing neurons in rat spinal cord. J Neurosci 26:1833-1843.

Traub RJ, Mendell LM (1988) The spinal projection of individual identified A-delta- and C-fibers. J Neurophysiol 59:41-55.

Woodbury CJ, Koerber HR (2007) Central and peripheral anatomy of slowly adapting type I low-threshold mechanoreceptors innervating trunk skin of neonatal mice. J Comp Neurol 505:547-561.

Woodbury CJ, Ritter AM, Koerber HR (2001) Central anatomy of individual rapidly adapting low-threshold mechanoreceptors innervating the "hairy" skin of newborn mice: early maturation of hair follicle afferents. J Comp Neurol 436:304-323.

Yaksh TL (1989) Behavioral and autonomic correlates of the tactile evoked allodynia produced by spinal glycine inhibition: effects of modulatory receptor systems and excitatory amino acid antagonists. Pain 37:111-123.

Yasaka T, Kato G, Furue H, Rashid MH, Sonohata M, Tamae A, Murata Y, Masuko S, Yoshimura M (2007) Cell-type-specific excitatory and inhibitory circuits involving primary afferents in the substantia gelatinosa of the rat spinal dorsal horn in vitro. J Physiol 581:603-618.

Yoshimura M, Nishi S (1995) Primary afferent-evoked glycine- and GABAmediated IPSPs in substantia gelatinosa neurones in the rat spinal cord in vitro. J Physiol 482:29-38. 